\title{
UMA PROPOSTA DE VERIFICAÇÃO AUTOMÁTICA DO PROCESSO DE AVALIAÇÃO MÚTUA EM ESTUDOS COLABORATIVOS
}

\author{
JOÃO PESSOA/PB MAIO/2018 \\ Clauirton Siebra - UFPB - clauirton@ci.ufpb.br \\ Anielton Nascimento - UFPB - anieltonjn@ppgi.ci.ufpb.br \\ Ellen Cruz - UFPB - cruzedn@gmail.com \\ Marismar Costa - UFPB - cmarismar@gmail.com \\ Tipo: Relato de Experiência Inovadora (EI) \\ Categoria: Métodos e Tecnologias \\ Setor Educacional: EDUCAÇÃO SUPERIOR
}

\begin{abstract}
RESUMO
Este trabalho utiliza conceitos de avaliação mútua, integrados a elementos de gamificação, como forma de motivar a colaboração de estudantes em pequenos grupos de estudo. Uma das principais contribuições do trabalho é o desenvolvimento de um processo de avaliação mútua automático, o qual pode ser integrado ao processo de avaliação mútua feita pelos alunos, de modo que os resultados das avaliações sejam mais confiáveis. A proposta foi utilizada em uma das turma do curso de Licenciatura em Computação, modalidade a distância. Os resultados mostram que, além de garantir uma maior segurança no processo de avaliação, também é possível verificar grandes discrepâncias neste processo, assim como gerar diferentes formas de comparação entre o desempenho de alunos e grupos de alunos, considerando diferentes atributos propostos neste trabalho.
\end{abstract}

Palavras-chave: Ensino a Distância, Participação Online, Avaliação Mútua, Gamificação 


\section{1 - Introdução}

A alta taxa de evasão é um dos principais problemas enfrentados na educação a distância. De fato, diversos trabalhos na área de Informática na Educação (AMERI et al., 2016; KOSTOPOULOS et al., 2017; FERNANDES et al., 2016) discutem este problema e propõe soluções para atenuar o mesmo. Uma alternativa que vem sendo utilizada para reduzir esta evasão é baseada em estudos educacionais e psicológicos (DILLENBOURG et al. 1995), os quais evidenciam o benefício das interações entre os alunos para a aprendizagem e assimilação natural do conteúdo (BLOM et al., 2013). Neste contexto, o desenvolvimento do autoaprendizado é um fator importante que surge por meio do entrosamento do alunato e contribui para habilidades como o pensamento crítico, a autorreflexão e co-construção de conhecimento.

O estudo da aprendizagem colaborativa ou em grupos já é tema de diversos trabalhos. Em seu artigo sobre como definir, medir e melhorar a colaboração (SOPHIE e PHILIP, 2005), por exemplo, os autores concluíram que a colaboração seria reforçada se os usuários tivessem ao seu dispor ferramentas mais intuitivas, fáceis de compreender e usar. Ao dispormos dessas ferramentas, temos como facilitar o processo de aprendizagem através da participação e colaboração dos alunos com os próprios alunos e com os instrutores. Nessa perspectiva, Brindley et al (2009) afirma que as plataformas virtuais de aprendizagem oferecem um ambiente propício para a aprendizagem social através do suporte à interação dos alunos, o que inclui possíveis processos de avaliação mútua como forma de motivar a participação de tais alunos.

A proposta deste trabalho é o desenvolvimento de um modelo automático que dê mais confiabilidade ao Processo de Avaliação Mútua (PAM). Este modelo estará associado ao uso de técnicas de 'gamification' e será utilizado com pequenos grupos de alunos dentro da plataforma Moodle. O objetivo final é motivar a participação e contribuição ao longo de atividades colaborativas, de modo que haja a construção compartilhada do conhecimento entre os alunos na plataforma.

\section{2 - Referencial Teórico}

De acordo com Guijarro (2000), o processo de avaliação mútua analisa a forma como os indivíduos reciprocamente regulam a aprendizagem cognitiva de outros processos e envolvem-se em modos comuns de regulação cognitiva. Sob essa ótica, estaríamos trabalhando com indivíduos que sabem o que querem e, participando de um processo de aprendizagem, seriam convidados a avaliar a si mesmo e a outros no decorrer do processo de aprendizagem (JAUME e NEUS, 2003). Ao analisarmos o trabalho de 
Guijarro (2000), concluímos que a autora traz consigo a percepção que o Processo de Avaliação Mútua é importante para a educação porque, ao inserir os alunos em ambientes sociáveis dentro da sala de aula, tais como pequenos grupos de trabalho, estamos formalizando uma das mais difíceis tarefas educacionais. Para ela, o Processo de Avaliação Mútua corrobora as estruturas educacionais ao inserir os alunos no ambiente de vida na sociedade contemporânea. De forma similar, Perrenoud (2000) entende que o Processo de Avaliação Mútua é importante para o fortalecimento das estruturas educacionais pois, ao inserir o aluno no processo de avaliação, esse se sentirá motivado a contribuir para ampliar as relações educacionais existentes.

Para Shiba e Sugawara (2014), "a percepção de avaliação mútua do trabalho é mais fácil e mais precisa quando é limitada aos membros do mesmo grupo porque os membros do grupo têm testemunhado as atividades dos outros alunos desse grupo". Isso nos leva a concluir que o trabalho em grupos que se autorregulam (avaliam), tende a produzir melhores resultados, como também cada elemento do grupo tende a melhorar no cumprimento de suas atividades. Dentro do grupo, a regulação mútua atua de forma a fazer com que o participante se auto avalie e, consequentemente, obtenha respostas para as suas dúvidas com maior rapidez. Dessa forma, o processamento das informações e o acompanhamento do estudo serão mais fieis a cada grupo, obedecendo às particularidades e resolvendo problemas como a demora em obter o feedback dos demais participantes. Sob essas condições, entende-se que "o grupo monitora a sua compreensão em curso em um processo onde os alunos mutuamente regulam a sua aprendizagem conjunta" (Guijarro, 2000).

Porém, devemos ter cuidado ao utilizar o processo de avaliação mútua, haja vista que alguns alunos podem avaliar de forma desonesta, aumentando ou diminuindo a avaliação do seu parceiro. Para diminuir essa possibilidade, nosso trabalho foi proposto de forma que, mesmo que os grupos permaneçam os mesmos do início ao fim de cada disciplina, possa haver um cálculo à parte para validar a percepção dos alunos. Esse cálculo é feito através do Processo Automático de Avaliação Mútua (PAMA), o qual é o principal tema deste trabalho.

\section{3 - Procedimentos Metodológicos}

O processo de avaliação mútua tradicional é composto por duas notas. A primeira corresponde ao processo de se autoavaliar; enquanto a segunda se refere à média das avaliações recebidas por cada aluno. Partimos do pressuposto que cada integrante do grupo deve se avaliar, nas competências elencadas, além de avaliar os outros integrantes. Deste modo é gerada uma rede com um conjunto de avaliações para cada 
participante em cada competência.

Um dos requisitos para que o processo de avaliação mútua ocorra de forma satisfatória é a definição do que deve ser avaliado de fato no processo. Sendo assim, após a revisão literária e uma análise prévia do que se deseja com o ensino a distância, foram definidas as cinco competências descritas a seguir:

- Participação na sala de aula virtual: define o quão assíduo o aluno é, se ele acessa regularmente a sala de aula, faz as atividades e participa do curso;

- Participação nos fóruns e chats: define o quão o aluno é participativo nos grupos de discussão, fóruns e chats, como ferramentas de fomento a discussão;

- Contribuição efetiva: define o quão o aluno contribuiu para o desenvolvimento do curso, através das discussões nos fóruns e envio de perguntas;

- Envio das atividades: define o quão o aluno é responsável e se ele enviou as atividades obrigatórias e/ou opcionais;

- Nota obtida nas atividades: avalia se o aluno enviou as atividades, buscando-se saber se ele as enviou apenas para cumprir suas obrigações ou se ele efetivamente realizou as atividades.

Para se chegar ao processo de avaliação mútua, foi definido que, em cada uma dessas competências, a avaliação irá obedecer uma escala de 1 a 5 pontos, definida da seguinte forma: 1 (péssimo), 2 (ruim), 3 (razoável), 4 (bom) e 5 (muito bom). A composição do conceito do aluno em cada competência será automaticamente calculada a partir do sistema proposto (PAMA). Porém, vejamos primeiro como ocorre o processo de avaliação manual (PAM). Neste processo, o conceito é obtido através de uma média simples em que $50 \%$ é a nota da autoavaliação que cada aluno faz de si. Os outros $50 \%$ da nota é obtido a partir da média das notas que o aluno recebeu dos demais colegas. A equação abaixo mostra como é calculada a nota de cada conceito do aluno que recebe mais de uma avaliação no processo de avaliação mútua:

$$
\bar{X} C_{Z}=N A A C_{Z} * 0,5+\frac{1}{N} \sum_{i=1}^{n} f C_{Z}(i) * 0,5
$$

Nesta equação temos que:

- $\mathbf{X C}_{\mathbf{Z}}$ : corresponde à média obtida na competência $z$;

- NAACZ: corresponde à nota da autoavaliação do aluno na competência $z$;

- Somatório: corresponde ao cálculo da média aritmética de $n$ conceitos recebidos pelo aluno na competência $z$. 
De acordo com o sistema proposto, o processo de avaliação mútua define uma pontuação do aluno para cada competência avaliada e essa pontuação será confrontada com a pontuação que internamente o sistema irá calcular (PAMA). Ao final, a pontuação do aluno em cada competência será uma média ponderada entre a pontuação obtida no processo de avaliação mútua e a nota calculada pelo sistema. Compete ainda informar que a ponderação será definida pelo tutor/professor de cada disciplina. Examplos de ponderação são (90\% PAM, 10\% PAMA) ou (50\% PAM e 50\% PAMA).

Com os dados obtidos e processados, o aluno terá como acompanhar o seu desenvolvimento na disciplina, comparando o seu rendimento no grupo e na turma, obtendo uma pontuação que será sempre atualizada à medida que a disciplina evolui. Durante este processo de avaliação mútua, alguns elementos de 'Gamification' foram adotados. O primeiro elemento foi um sistema de pontuação para cada aluno. Através desse sistema de pontuação, podemos criar um 'rank' enumerando a posição do aluno dentro da disciplina, estimulando, dessa forma, o aluno a sempre se superar.

O segundo elemento adotado foi a existência do feedback imediato. Ao cumprir uma etapa como o envio de uma atividade, por exemplo, ou uma participação em um fórum, o aluno tem sua pontuação atualizada e pode conferir os pontos obtidos pela realização daquela etapa. Outro elemento do 'Gamification' empregado foi o sistema de recompensa, em que ao final de uma etapa da disciplina, os alunos podem receber pontos. Essa pontuação será a critério do tutor/professor, mas pode ser obtida através do 'rank' criado. Além disso, ao final da disciplina, serão ofertadas medalhas comumente chamada de 'badges' no ambiente virtual. Convém ressaltar ainda que os elementos aqui citados são os mais básicos encontrados em qualquer sistema que adote a gamificação. Por justamente serem os mais básicos, são os mais utilizados e de fácil entendimento da sua rotina de funcionamento.

De acordo com o que foi enumerado, o Processo de Avaliação Mútua, utilizando conceitos do 'Gamification', irá disponibilizar aos alunos, professores e tutores; novos meios de se verificar o andamento da realização das atividades, além de esboçar um gráfico informando o desempenho do aluno de forma individual, comparando com o grupo e a turma. Os gráficos a serem disponibilizados seriam construídos a partir da realização do PAM e do PAMA, obedecendo os procedimentos metodológicos descritos neste trabalho.

A Figure 1 mostra a existência de cinco eixos, cada um correspondendo a uma competência elencada no Processo de Avaliação Mútua, junto com a escala de avaliação de cada competência de acordo com a Média. As cores são para diferenciar o 
desempenho do aluno (vermelho) em comparação com o grupo (azul) e a turma (verde). A vantagem deste tipo de gráfico é a facilidade em se realizar comparações entre estes diferentes níveis de desempenho, considerando as cinco competências.

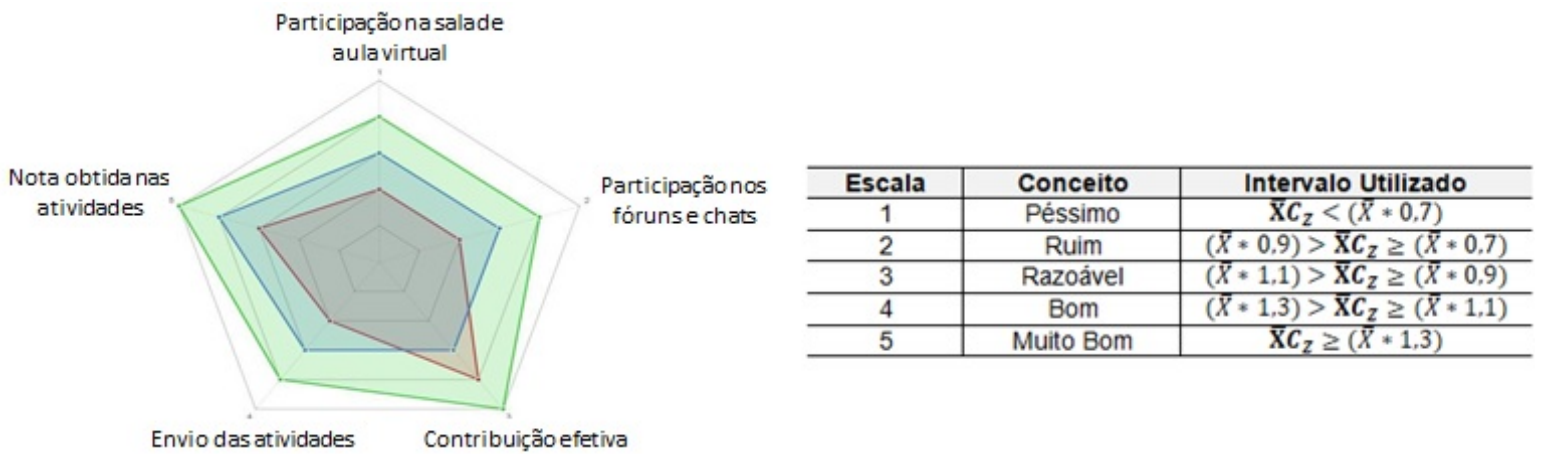

Figura 1: Modelo de gráfico disponibilizado para alunos, professores e tutores e a escala de avaliação de cada competência de acordo com a Média.

\section{4 - Resultados e Discussão}

O Processo de Avaliação Mútua Automática foi aplicado na disciplina de Banco de Dados do curso a distância de Licenciatura em Computação, a qual contou com 8 alunos que cursaram a disciplina durante 19 semanas de aula. Para realizarmos o PAMA, foi necessário responder às perguntas das competências elencadas. Nesse processo, buscamos também distinguir cada ação realizada pelo aluno. Ao realizamos a consulta ao banco de dados, obtivemos, para cada aluno, um arquivo com os seus respectivos acessos. Nessa consulta, os dados foram separados e tabulados de tal forma a obter a informação necessária para responder aos questionamentos das competências. Esses resultados obtidos correspondem aos dados de participação dos alunos de acordo com a atividade.

A partir da tabulação dos dados referenciados e da obtenção da informação de acesso dos alunos, foi possível realizar o cálculo de algumas medidas de desempenho, as quais podem ser observadas na Tabela 1. Alguns dados nos chamam a atenção, como é o caso do alto índice do Coeficiente de Variação da atividade do tipo chat, além do alto grau da Variância de todos os acessos dos alunos.

A partir dos dados da Tabela 1, podemos obter algumas conclusões. A primeira é que a turma não é muito homogênea com relação aos acessos, uma vez que ocorre um alto índice do Coeficiente de Variação medido. A segunda conclusão é que a atividade do tipo chat é pouco utilizada, fato esse que diminui a proximidade dos alunos com 0 professor e tutor da disciplina. 
Tabela 1. Relação de acesso dos alunos por medidas de desempenho

\begin{tabular}{l|r|r|r|r}
\hline \multirow{2}{*}{ Medidas de Desempenho } & \multicolumn{4}{|c}{ Acesso por Atividade } \\
\cline { 2 - 5 } & \multicolumn{1}{|c|}{ Arquivo } & \multicolumn{1}{c}{ Fórum } & \multicolumn{1}{c}{ Tarefa } & \multicolumn{1}{c}{ Chat } \\
\hline Variância & 333,27 & $21.159,41$ & $6.704,00$ & 287,41 \\
\hline $\begin{array}{l}\text { Desvio-Padrão } \\
\begin{array}{l}\text { Coeficiente de } \\
\text { Variação }\end{array}\end{array}$ & 56,83 & 145,46 & 81,88 & 16,95 \\
\hline
\end{tabular}

De posse dos dados de acesso dos alunos tabulados, obteve-se a pontuação de cada aluno para as duas primeiras competências elencadas (participação na sala de aula virtual e participação nos fóruns e chat). Para se obter esse resultado, utilizou-se como medidas de desempenho a média e o desvio padrão calculados anteriormente. Foram definidas como métrica a média de acesso dos alunos de acordo com o tipo de atividade proposta. Dessa forma, conseguiu-se montar uma fórmula que calculasse o conceito de cada aluno dentro das competências.

Para a obtenção dos dados da terceira competência, as participações efetivas dos alunos nos fóruns e chats foram contabilizadas e marcadas como positivas, utilizando um processo humanizado nessa consulta. Ao final dessa contabilização, foram calculadas as medidas de desempenho e obtidos os conceitos finais. A obtenção dos dados para definição do conceito do aluno na Competência 4 também foi realizada de forma manual, buscando na lista de alunos quais atividades eles haviam entregue. Vale salientar que na disciplina em questão existem dois grupos de atividades online: as atividades obrigatórias e as atividades opcionais. O primeiro grupo totalizou 12 atividades, enquanto que o segundo 7 atividades.

Para a obtenção dos dados para a avaliação da Competência 5, foi elaborada uma tabela com o percentual de acerto de cada aluno por atividade. Nesse sentido, foram contabilizadas as atividades obrigatórias, as opcionais e também as provas presenciais. Como cada atividade possui uma distribuição de pontos diferentes para a contabilização do percentual de acerto (obrigatórias e opcionais), foi necessário o uso de uma tabela auxiliar que relacionasse os pontos obtidos naquela atividade e depois os transformassem em percentuais de acerto dentro da faixa de cada atividade.

Para as atividades online foram aferidos os percentuais de acerto de acordo com cada atividade. Depois realizou-se o cálculo da média de acertos dessas atividades, produzindo assim uma tabela final contendo o percentual de acertos de alunos por tipo de atividade e a média de acertos desses alunos. De posse dos conceitos obtidos para 
cada aluno, foram realizados alguns testes com a finalidade de obter resultados sobre 0 modelo proposto. Após todos os cálculos realizados, chegamos a seguinte relação que contém todos os conceitos dos alunos nas cinco competências discutidas: Aluno 01 $(3,5,3,5,4)$, Aluno $02(5,3,5,4,5)$, Aluno $03(1,1,1,1,1)$, Aluno $04(5,5,3,4,2)$, Aluno 05 $(5,5,1,5,2)$, Aluno $06(1,1,1,1,1)$, Aluno $07(1,1,2,2,4)$ e Aluno $08(4,5,2,4,5)$. Com tais informações é possível detectar que dois alunos (Aluno 03 e Aluno 06) obtiveram um desempenho muito abaixo do esperado em todas as competências. Tal desempenho culminou consequentemente na reprovação direta por média dos alunos em questão. Da mesma forma, podemos observar que dois alunos (02 e 08) se destacaram de forma positiva, obtendo conceitos acima do esperado nas competências. $O$ fato de existir dois alunos com conceitos em todas as competências abaixo do esperado, em contraste com outros dois alunos que obtiveram conceitos elevados, culminou na obtenção de altos índices de variância em cada competência, conforme podemos observar na Tabela 2:

Tabela 2. Medidas de Desempenho da Turma

\begin{tabular}{l|r|r|r|r|r}
\hline \multirow{2}{*}{ Medidas de Desempenho } & \multicolumn{5}{|c}{ Acesso por Atividade } \\
\cline { 2 - 6 } & \multicolumn{1}{|c|}{1} & \multicolumn{1}{c}{2} & \multicolumn{1}{c}{3} & \multicolumn{1}{c}{4} & \multicolumn{1}{c}{5} \\
\hline Média & 3,13 & 3,25 & 2,25 & 3,25 & 3,00 \\
\hline Variância & 3,554 & 3,929 & 1,929 & 2,786 & 2,857 \\
\hline Desvio-padrão & 1,885 & 1,982 & 1,389 & 1,669 & 1,690 \\
\hline $\begin{array}{l}\text { Coeficiente de } \\
\text { Variação }\end{array}$ & 60,323 & 60,987 & 61,721 & 51,355 & 56,344 \\
\hline Erro padrão da média & 0,666 & 0,701 & 0,491 & 0,590 & 0,598
\end{tabular}

Nos chama a atenção o Coeficiente de Variação das três primeiras competências, estando todos acima de 60 . Como as três primeiras competências avaliam a participação do aluno na disciplina, podemos concluir que a falta de interação dos alunos pode ter sido um fator de reprovação deles. A média do desempenho em relação à turma como um todo pode ser visualizada no gráfico seguinte (Figura 2a). Como o nosso projeto pretende trabalhar com pequenos grupos, os 8 alunos foram divididos em dois grupos ("A" e "B") de 4 participantes cada. A Figura $2 b$ mostra o desempenho do grupo "A". A comparação do desempenho do grupo A com o da Turma indica que a média do grupo foi superior em quatro das cinco competências avaliadas. Como exemplo de avaliação automática do aluno, podemos observar o desempenho do Aluno 02 que, de acordo com os dados analisados, obteve o melhor desempenho na Turma.

Nosso objetivo é que os alunos estejam conscientes da sua participação no processo e se atentem as competências que eles precisam melhorar. Gráficos como este podem 
ajudar nesta autoanálise, além de favorecer a comparação do indivíduo com o todo. Como cada aluno está avaliando seus pares e sendo avaliado pelos mesmos, tal processo tente a motivar a melhora individual de cada participante.

(a)

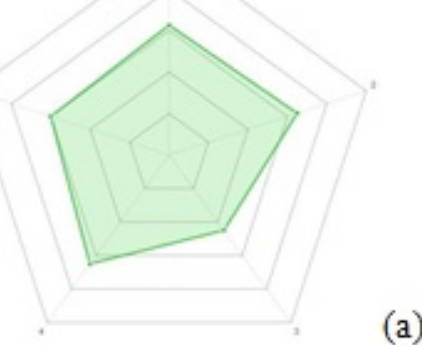

(b)

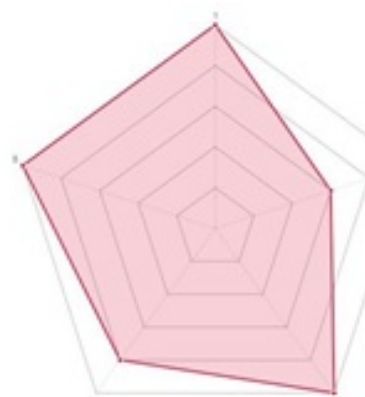

(c)

Figura 2: Desempenho de um dos grupo (a) da turma, da própria turma (b) e de um dos alunos desta turma (c).

\section{5 - Conclusão e Trabalhos Futuros}

Esta pesquisa teve como objetivo propor uma maneira automática de se verificar o processo de avaliação mútua que ocorre em um ambiente de educação a distância. Este tipo de avaliação automática é importante porque a avaliação manual, realizada pelos próprios alunos, pode conter algum viés devido a questões pessoais entre eles. Juntos, o Processo de Avaliação Mútua (PAM) e Processo de Avaliação Mútua Automática (PAMA) podem compor um resultado final mais fiel, o qual pode ser visualizado em gráficos que facilitem comparações das competências pelos próprios alunos. Resultados iniciais mostram que realmente existe uma diferença significante entre os as duas formas de avaliação. Trabalhos futuros tem o objetivo de formalizar esta análise quantitativa e também realizar uma análise qualitativa para o entendimento desta diferença. Um possível problema é que a quantificação automática das competências esteja descalibrada, o que geraria resultados incorretos. Outra possibilidade é que realmente 0 aspecto pessoal de relação entre os alunos esteja enviesando as avaliações. Além disso, é necessário aumentar o tamanho da nossa amostra. De fato, o uso de apenas 8 alunos é uma limitação deste trabalho. Porém, este estudo piloto foi importante para o ajuste da quantificação das competências.

\section{Referências}

AMERI, S., FARD, M. J., CHINNAM, R. B., REDDY, C. K. Survival analysis based framework for early prediction of student dropouts. Proceedings of the 25th ACM International on Conference on Information and Knowledge Management, pp. 
903-912, 2016.

BLOM, J., VERMA, H., LI, N., SKEVI, A. MOOCs are More Social than You Believe" eLearning Papers, n. 3, 2013.

BRINDLEY, Jane; BLASCHKE, Lisa Marie; WALTI, Christine. Creating Effective Collaborative Learning Groups in an Online Environment. The International Review of Research in Open and Distributed Learning, [S.I.], v. 10, n. 3, jun. 2009.

DILLENBOURG, P., BAKER, M. J., BLAYE, A., O'Malley, C. The evolution of research on collaborative learning. Learning in Humans and Machine: Towards an interdisciplinary learning science, pp. 189-211, 1995.

FERNANDES, A., SILVA, D., SIEBRA, C. Modelo de Apoio para Desenvolvimento de Objetos de Aprendizagem Utilizando Técnicas de RPG. Anais do 22 CIAED Congresso Internacional de Educação a Distância. Pp. 1-10, 2016.

GUIJARRO, R. B. Inclusive education in Latin America. Meeting special and diverse educational needs: Meeting special education a reality. Helsinki, Finland: Ministry of Foreign Affairs, pp. 40-51, 2000.

Jaume, J., Neus, S. A função pedagógica da avaliação. Avaliação como apoio à aprendizagem. Porto Alegre: Artmed, pp. 23-45, 2003.

KOSTOPOULOS, G., KOTSIANTIS, S., RAGOS, O., GRAPSA, T. N. Early dropout prediction in distance higher education using active learning, Proc. of the 8th Int. Conference on Information, Intelligence, Systems \& Applications, pp. 1-6, 2017.

PERRENOUD, P. 10 novas competências para ensinar. Artmed editor, 2000.

SHIBA, T. SUGAWARA. Fair assessment of group work by mútual evaluation based on trust network. Frontiers in Education Conference, pp. 1-7, 2014.

SOPHIE, H., PHILLIP, K. Defining, Measuring, and Improving Collaboration though Evaluating the Cornell Library Collaborative Learning Computer Laboratory. unpublished Cornell class report, 2005. 